\title{
ADAPTATIONS DE LA MÉLODIE AUX VARIATIONS DE LONGUEUR DU VERS AUX XII ${ }^{E}$ ET XIII ${ }^{E}$ SIÈCLES
}

\section{ADJUSTMENT OF TUNE TO VARYING LINE LENGTH IN $12^{\text {TH }}$ AND $13^{\mathrm{TH}}$ CENTURY FRENCH MUSIC}

\section{ADAPTACIONES DE LA MELODÍA A LAS VARIACIONES DE LONGITUD DEL VERSO EN LOS SIGLOS XII Y XIII}

\author{
TimothéE Premat \\ Université Paris 8 \\ \& \\ Sophie ChOUvion \\ Université Lyon 2
}

Résumé: Cette étude est dédiée à la description et à la formalisation des mécanismes qui permettent d'adapter une mélodie à des vers dont la longueur varie. Notre corpus est constitué de pièces de musique vocale écrites en français aux $\mathrm{XII}^{\mathrm{e}}$ et $\mathrm{XIII}^{\mathrm{e}}$ siècles, qui sont parmi les plus anciennes pièces vernaculaires à nous être parvenues accompagnées de leur notation musicale. Il s'agit donc d'étudier les modifications que subit la mélodie pour s’adapter à la variation de longueur du vers, tout en conservant un sentiment d'équivalence entre les différentes versions de la mélodie. 
Mots-clés: musication, trouvères, équivalence, mélodie, métrique, coupe épique, rime féminine.

Abstract: This study aims at describing and analyzing mechanisms that allow a tune to be sung with lines of different length. Our corpus comprises French songs from the $12^{\text {th }}$ and $13^{\text {th }}$ centuries, which are among the earliest French vernacular songs that have been preserved along with their musical notation. Our study examines the way these songs adjust the tune to the line length variation, without breaking the equivalence relation between the different versions of the tune.

Keywords: textsetting, trouveres, equivalence, tune, metrics, epic break, feminine rime.

Resumen: Este estudio está dedicado a la descripción y formalización de los mecanismos que permiten adaptar una melodía a versos de diversa extensión. Nuestro corpus se compone de piezas de música vocal, escritas en francés en los siglos XII y XIII y encontradas entre las piezas vernáculas más antiguas que nos han llegado acompañadas de su notación musical. Se trata, pues, de estudiar las modificaciones que sufre la melodía para adaptarse a la variación de la longitud del verso, manteniendo un sentimiento de equivalencia entre las distintas versiones de la melodía.

Palabras clave: musicación, trouvères, melodía, métrica, corte épico, rima femenina. 


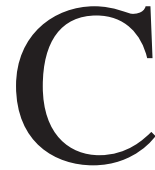

ette étude se fonde sur l'examen d'un corpus de chansons médiévales issues de la production des trouvères (à partir du XII ${ }^{\mathrm{e}}$ siècle), d'Aucassin et Nicolette (fin XII ${ }^{\mathrm{e}}$ - déb. XIII ${ }^{\mathrm{e}}$ siècle) et de Li Gieus de Robin et de Marion (XIII ${ }^{\mathrm{e}}$ siècle) ${ }^{1}$. Nous visons à documenter et analyser, dans l'approche générative de l'interface entre musique et texte, les processus d'adaptation de la musique aux variations de longueur du texte. Nous nous intéressons particulièrement à la façon dont ces processus permettent le maintien d'un certain degré d'homologie entre deux segments musicaux pourtant non identiques ${ }^{2}$.

Nous appelons musication (équivalent de l'anglais textsetting) l'approche générative de l'interface entre musique et texte. Cette approche se fonde sur le fait que, alors que la musique déploie des stratégies de composition extrêmement diversifiées, il existe des principes récurrents dans la façon dont sont associées les unités du texte et celles de la musique, principes qui se traduisent par des intuitions chez les compositeurs, les chanteurs et les auditeurs ${ }^{3}$. Les paramètres de l'application de ces principes dépendent non seulement du type de musique auquel le sujet est exposé et des grammaires d'association entre langue et musique qu'applique cette musique, mais également des propriétés phonologiques du langage qui est chanté. C'est en effet le degré d'homologie dépendent donc non seulement du type de musique auquel le sujet est exposé et des grammaires d'association entre langue et musique qu'applique cette musique, mais également des propriétés phonologiques du langage qui est chanté. C’est le degré d’homologie entre les éléments musicaux et les éléments linguistiques qui semble déclencher des contraintes sur leur association ${ }^{4}$. Ainsi, l'emphase a

${ }^{1}$ Nous avons toujours travaillé à partir des sources manuscrites.

2 Dans cet article, nous donnons à identique un sens restreint : si A est identique à $\mathrm{A}$ ', alors $A=A^{\prime}$, mais si $A \simeq A^{\prime}$, alors $A$ et $A^{\prime}$ ne sont pas identiques.

${ }^{3}$ Halle, John, \& Lerdhal, Fred, « A Generative Textsetting Model », Current Musicology, 55 (1993), pp. 3-23, p. 3.

${ }^{4}$ Dell, François, \& Halle, John, « Comparing musical textsetting in French and English songs ", en Jean-Louis Aroui, Andy Arleo (eds.), Towards a Typology of poetic forms, 
principalement été mise sur le lien entre les structures rythmiques du langage et celles de la musique, dans une acception restreinte de ce qu'est le rythme. Pourtant, la musication pose également des problèmes d'ordre mélodique, c'est-à-dire relatifs à la succession des changements de hauteur de note. En effet, lorsque la musique comporte des structures itératives, la musication doit assurer un certain degré d’homologie entre les différentes instanciations du même segment musical, de manière à ce que ces instanciations soient perçues comme équivalentes (à défaut d’être identiques), c'est-à-dire qu'elles aient même valeur structurale et puissent commuter entre elles au sein d'une même structure.

\section{Identité, homologie et relation d'équivalence}

Dans de nombreux répertoires chantés, il arrive qu’une même séquence de notes doive être associée à des segments linguistiques dont le nombre de syllabes varie. Cela est particulièrement vrai lorsque la musique dispose d'une itération strophique (les mêmes séquences de notes sont répétées sur différentes strophes), mais peut aussi se produire à l'intérieur même des strophes, des vers, voire en dehors de toute structure strophique. Dans notre corpus, les variations du nombre de syllabes à chanter sur une même séquence de notes se situent à la césure (coupe épique : il y a un - $e$ extramétrique à la césure $)^{5}$ et en fin de vers (terminaison féminine, communément appelée rime féminine bien que ce ne soit pas la qualité de rime qui soit ici en jeu).

Lorsqu'il y a itération d'une même séquence de notes, il y a production d'une structure (p.ex. ABA'B') et celle-ci demande à être perçue. Pour que ce soit le cas, le lecteur/auditeur doit être à même de reconnaître un lien de parenté entre A et A' et entre B et $B$ '. Cette reconnaissance repose sur l'identification de propriétés communes entre A et A' et entre B et B'. Lorsque A et A' sont identiques, la relation d'homologie est totale et donc suffisante. Mais, lorsque $\mathrm{A}$ et $\mathrm{A}^{\prime}$ disposent de divergences, ces divergences doivent être restreintes et licites pour que A' soit perçu comme résultant d'une itération de A. Le même principe existe en métrique 'lit-

from language to metrics and beyond, Amsterdam/Philadelphia, John Benjamins Publishing Company, 2009, pp. 63-78, p. 64.

5 Voir définition dans Billy, Dominique, «Théorie et description de la césure : quelques propositions », en Furio Brugnolo, Francesca Gambino (eds.), La lirica romanza del Medioevo, Storia, tradizioni, interpretazioni, Padoue, Unipress, 2009, vol. I, pp. 385423. 
téraire' (c'est-à-dire non chantée), et peut être décrit comme une équivalence ${ }^{6}$ de surface :

1. Équivalence. La relation qui unit deux segments A et A' est une relation d'équivalence si A et A' partagent des propriétés structurales suffisantes à l'identification intuitive par le lecteur/auditeur d'une relation d'homologie entre A et A'.

Cette notion d'équivalence est essentielle à notre approche. Il importe de considérer que A et A' ne sont en réalité jamais exactement identiques, d'une part parce que A' arrive après A, et d'autre part parce que seul un programme informatique MIDI est capable de réaliser deux fois exactement de la même manière le même segment musical. De ce fait, même lorsque A et A' sont identiques (toutes leurs propriétés sont les mêmes), ils ne sont en réalité pas identiques mais sont évidemment équivalents, puisque leur homologie est parfaite. Une relation d'équivalence peut également réunir A et A' lorsque certaines propriétés de A ne se retrouvent pas dans A', à condition que d'autres propriétés leur soient communes et que ces propriétés soient suffisantes à l'établissement d'une forte homologie. Notre étude vise à définir, dans un corpus de musique vocale en langue d'oïl des $\mathrm{XII}^{\mathrm{e}}$ et XIII ${ }^{\mathrm{e}}$ siècle, quelles sont les propriétés suffisantes à l'établissement de cette relation d'équivalence entre segments musicaux non identiques.

Dans la structure d'une pièce de musique vocale, des segments musicaux équivalents doivent pouvoir commuter entre eux. François Dell et John Halle ont montré que, pour que deux séquences de notes puissent commuter au sein de la même structure, elles doivent respecter le même contour mélodique 7 . Ce qu'ils désignent par contour mélodique correspond uniquement à la succession des changements de hauteur de note, et représente l'une des dimensions de la structure fondamentale d'un segment musical. Ainsi, le segment musical (a) sol la si do si la la sol sol peut être réduit à un contour mélodique (b) sol la si do si la sol. De ce fait, (a) peut

\footnotetext{
${ }^{6}$ Nous empruntons le mot à DE Cornulier, Benoît, Art Poëtique, Notions et problèmes de métrique, Lyon, Presses Universitaires de Lyon, 1995, pp. 21-32, qui le reçoit luimême de Jakobson. Chez Jakobson, ce principe permet de convertir les événements linguistiques en propriétés comparables et commensurables, ce qui représente l'essence de la fonction poétique du langage : «The poetic function projects the principle of equivalence from the axis of selection into the axis of combinaison. Equivalence is promoted to the constitutive device of the sequence. " (JAKOBSON, Roman, « Linguistics and Poetics », en Thomas A. Sebeok (ed.), Style in Language, New York/London, MIT Press/John Wiley \& Sons, 1960, pp. 350-377, p. 358 ; italiques de l'auteur).

7 Dell, François, \& Halle, John, " Comparing musical textsetting ... », cit., p. 69.
} 
être modifié dans une certaine mesure sans que cela ne modifie (b). Ainsi, (a') sol la si do si la la sol sol sol a le même contour mélodique (b) que (a). Si c'est le respect de (b) qui définit la relation d'équivalence, alors (a) et (a') sont équivalents. Cette situation est illustrée dans la musique associée au premier hémistiche des vers 1-3 de la chanson "En un vergier », en Figure 1. Pour adapter la musique à la présence d'une syllabe supplémentaire (le -de de l'onde au v. 2), le manuscrit dispose d'une note de plus au v. 2, associée à cette syllabe supplémentaire et extramétrique (cet exemple est discuté en détail plus bas). De fait, la musique des vers 1 et 3 (a) partage bien le même contour mélodique que la musique du vers 2 (a'), ce qui maintient l'équivalence entre (a) et (a').

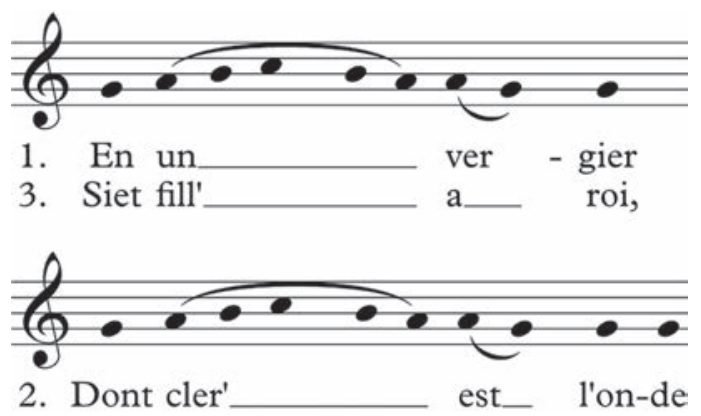

Figure 1. «En un vergier », vv. 1-3, premier hémistiche.

Nous montrerons plus bas que si la conservation du contour mélodique est une condition nécessaire à l'équivalence, elle n'est pas une condition suffisante. Pour l'instant, contentons-nous de l'approximation suivante :

2. Conservation du contour mélodique. Pour que deux segments musico-textuels A et A' soient perçus comme équivalents, ils doivent avoir le même contour mélodique.

Ce principe étant provisoirement exprimé ainsi, nous allons tester son application à des répertoires de musique médiévale de langue d'oïl des XII et XIII ${ }^{\mathrm{e}}$ siècles.

\section{Exploiter les sources en musique médiévale}

Un certain nombre de difficultés s'opposent $a$ priori à l'étude de ces adaptations de la mélodie dans des répertoires de musique médiévale vernaculaire française. La première difficulté tient à la 
nature des sources, écrites, dont l'interprétation n'est pas toujours aisée, et sujette à ce que Paul Zumthor appelle la mouvance ${ }^{8}$ : tout comme les textes non chantés, les textes et les mélodies des chansons peuvent varier de manuscrit en manuscrit. La seconde difficulté tient au fait que, le plus souvent, la musique n'est écrite que pour la première strophe de la chanson ; nous n'utilisons donc pas les variations de longueur du vers qui ne se trouveraient pas dans la partie notée directement sous la musique. Lorsque plusieurs manuscrits sont présents, qu’ils notent la même mélodie mais que certains semblent ignorer la coupe épique dans la musique tandis que d'autres la réalisent, la comparaison des différents manuscrits témoigne bien d'une forme d'adaptation de la mélodie à la variation du texte ; lorsque ce n'est pas le cas, notre étude nécessite que le segment musico-textuel soit présent sur le manuscrit deux fois, une fois avec l'adaptation et une fois sans. La troisième difficulté tient à la rareté des variations de longueurs des vers dans cette tradition métrique. En effet, un vers français ne peut, sans être mal formé, comporter de syllabe surnuméraire qu'à son terme (terminaison ou rime féminine) et à la coupe (coupe épique). À cela s’ajoute la rigidité des formes du texte, qui n'autorisent la plupart du temps pas l'alternance libre des vers féminins et masculins, diminuant ainsi drastiquement nos chances de voir apparaître sous le même segment musical un vers masculin et un vers féminin. Enfin, s’y ajoutent la raréfaction objective des coupes épiques à partir de la production des trouvères, et le débat philologique à propos de leur existence et du besoin de les corriger ou non ${ }^{9}$.

Pour notre part, nous ne prenons pas de position sur cette question philologique : dans la mesure du possible, nous nous abstenons de corriger le texte ou la musique présents sur le manuscrit, et considérons que c'est bien la leçon du manuscrit, même divergente, qui représente nos données, et non l'état corrigé et parfait de l’œuvre qui précéderait la copie 'fautive' à laquelle nous avons accès. Ainsi, lorsqu'un manuscrit présente une coupe épique et que celle-ci est représentée dans la musique par une note correspondant au -e épique,

${ }^{8}$ Zumthor, Paul, Essai de poétique médiévale, Paris, Seuil, 1972, pp. 70-75.

${ }^{9}$ Pour un aperçu du débat relatif à l'existence de la coupe épique dans le répertoire des trouvères et à la difficulté de l'interprétation des sources pour valider ou confirmer cette hypothèse, voir notamment SANGIOvANNI, Fabio, « La cesura epica come problema lirico ", [ms. soumis à publication dans les actes des IV ${ }^{\text {èmes }}$ rencontres franco-italiennes Philologie et Musicologie (Morimondo/Pavie, 1-5/06/2019) dans la revue Textus \& Musica, soumis en 2020]. 
nous l'acceptons dans nos données, et ce, même lorsqu'elle se trouve devant une initiale vocalique, là où le philologue préfère souvent analyser une synalèphe plutôt qu'une syllabe extramétrique.

Une fois considérées toutes ces restrictions, l'examen de 157 chansons de trouvères ${ }^{10}$ ne nous a laissé qu'avec une vingtaine de cas analysables. Cela ne signifie pas que l'objet de notre analyse est trop rare pour être pertinent dans les chansons des trouvères, mais bien qu'il est caché par les sources, qui ne déploient pas la musique sur l'ensemble de la pièce. À cette vingtaine de cas s'ajoutent quelques cas provenant de Li Gieus de Robin et de Marion et d'Aucassin et Nicolette. Si nous n'exposons pas ici tous les cas recensés, ceux-ci sont toujours fortement cohérents avec les exemples que nous analysons.

Enfin, il importe de mentionner qu'il existe un débat sur la notation rythmique de nos répertoires. À l'exception de Li Gieus de Robin et de Marion, dans la plupart de nos manuscrits, le système graphique employé n’est rythmé que dans une certaine mesure : il indique des regroupements et des relations de dépendance (telle note est dépendante de telle autre note), mais n’indique pas de rythmes proportionnels exacts comme ceux de la musique moderne, où une blanche est égale à deux noires. Ainsi, sur nos manuscrits, les notes indépendantes sont toutes transcrites par le même signe, sans indication de durée, comme visible en Figure 2.

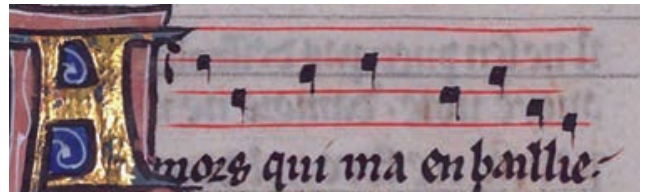

Figure 2. «Amors qui m’a en baillie » (ms. K, p. 365).

Certains musicologues s’autorisent à projeter des modes rythmiques sur cette musique, ce qui leur permet de réaliser des éditions modernes mesurées et précisément rythmées en termes proportionnels exacts ${ }^{11}$, tandis que d'autres préfèrent éditer cette musique en

${ }^{10}$ Ce sont les 157 chansons présentes avec musique notée dans l’ouvrage RosENBERG, Samuel N., Tischler, Hans, \& Grossel, Marie-Geneviève, Chansons des trouvères, Chanter m'estuet, Paris, Librairie générale française, 1995. Il est à noter que les éditeurs de cet ouvrage extrapolent eux-mêmes des adaptations de la musique au texte, souvent sans les signaler dans l'apparat critique.

${ }^{11}$ C'est par exemple le cas des éditeurs de Chansons des trouvères (cit.), qui éditent cet extrait dans une alternance de noires et de croches (p. 664). 
notes rondes, sans préciser ni mesure ni relation de proportionnalité exacte $^{12}$. En l'absence d'un consensus scientifique sur la question, nous adoptons l'approche minimaliste des éditions en notes rondes. De ce fait, nous ne sommes pas en mesure d'analyser les facteurs rythmiques de proportionnalité exacte des adaptations que nous documentons, ni leur relation avec la structure de temps faibles et forts de la musique (structure métrique chez Lerdhal \& Jackendoff ${ }^{13}$ ), ce qui a l'avantage paradoxal de nous forcer à nous concentrer uniquement sur la dimension mélodique de ces adaptations.

\section{Deux stratégies d'adaptation du matériel musical}

Dans nos chansons, nous observons deux stratégies d'adaptation du matériel musical qui conservent l'équivalence entre ses différentes itérations ${ }^{14}$ : la présence d'une note de plus dans le segment musico-textuel augmenté (doté de plus de syllabes) que dans le segment non augmenté et la présence d'une différence dans les ligatures constitutives des neumes, sans altération du nombre de notes. Dans ce qui suit, nous illustrons, décrivons et analysons chacune de ces stratégies. Avant de décrire ces deux stratégies, nous devons définir ce que nous entendons par note :

3. Note. Une note est un objet défini par les trois propriétés suivantes :

a. sa hauteur,

b. sa durée,

c. la position de son attaque au sein de la structure de temps forts et faibles de la musique.

En fonction des choix d'édition et d'analyse rythmique exposés plus haut, le paramètre (c) n’est pas utilisé dans nos analyses, tandis que le paramètre (b) ne nous concernera que marginalement. Par commodité, pour référer à une note donnée, nous utilisons sa hauteur comme étiquette. Par souci d'accessibilité, nous utilisons les sept noms de notes usuels en musique moderne.

$\overline{12}$ C'est par exemple le cas des éditeurs de Rosenberg, Samuel, N., Switten, Margaret, \& LE VoT, Gérard, Songs of the Troubadours and Trouveres: an anthology of poems and melodies, New York/London, Garland, 1998.

${ }^{13}$ Lerdhal, Fred \& JACKendoff, Ray, A Generative Theory of Tonal Music, Cambridge, MIT Press, 1987.

14 Nous avons également trouvé des cas où le segment musical n'est équivalent qu'avant la syllabe extramétrique, et est réécrit à partir de celle-ci de telle manière que l'équivalence ne saurait plus être assurée. Nous ne traitons pas ces cas. 
Ainsi, pour nous, la note est un objet qui précède la musication et qui est indépendant de la constituance neumatique et mélismatique. Un neume est constitué de plusieurs notes regroupées entre elles et est représenté dans la graphie par la continuité du trait de plume (ligature) ou par un enchaînement conventionnel de signes détachés ; dans nos transcriptions, nous rendons les neumes par des symboles de liaison. Un neume indique que les notes qui le composent doivent nécessairement être prononcées sur la même syllabe, mais les possibilités de mélisme (une seule syllabe chantée sur plusieurs notes) ne se réduisent pas aux seuls neumes : une même syllabe peut être chantée sur une seule note, sur plusieurs notes détachées, sur un seul neume, sur plusieurs neumes ou sur une combinaison de notes et de neumes. Nous considérons que la constituance des neumes et des mélismes n'est pas opaque pour la musication, dans la mesure où la musication est capable d'agir directement sur les notes qui les composent. Enfin, dans nos transcriptions, nous faisons apparaître les pliques, neumes spécifiques constitués d'une note principale et d'une note ornementale, d'importance structurale et rythmique inférieure, en indiquant cette dernière par une plus petite note.

Deux contraintes fondamentales de la musication veulent que toute syllabe linguistique $(\sigma)$ qui est prononcée ait besoin d'au moins une note sur laquelle être chantée, et qu'une note ne puisse recevoir qu'une seule syllabe ${ }^{15}$ :

4. $\sigma /$ NOTE. Toute syllabe prononcée doit être associée à au moins une note.

5. NotE/ $\sigma$. Une note doit être associée à une et une seule syllabe.

\section{III.1. Duplication}

L'une des stratégies d'adaptation du texte joue sur le nombre de notes du segment musico-textuel. Lorsqu'il y a contraste de nombre de notes entre deux segments musico-textuels équivalents, les notes qui sont ajoutées au segment le plus long sont le résultat d'une stratégie que nous appelons duplication. La duplication est la génération, à partir d'une note donnée, d'une ou plusieurs autres notes. La duplication ne change pas le contour mélodique lorsqu'elle recopie la hauteur de la note qui la précède (duplication progressive) ou de la note qui la suit (duplication régressive) ;

${ }^{15}$ Dell, François, « Text-to-tune alignment and lineation in traditional French songs », en Teresa Proto, Paolo Canettiri \& Gianluca Valenti (eds.), On the Association of Music and Lyrics in Sung Verse, Bern, Peter Lang, 2015, pp. 183-234, p. 192. 
l'orientation progressive ou régressive de la duplication ne peut être attestée que dans certains cas, comme en Figure 6 infra.

Dans la chanson « En un vergier », les vers 1, 2 et 3 sont chantés sur des segments musicaux équivalents, mais le v. 2 dispose d'une coupe épique devant initiale vocalique (la post-tonique de la coupe épique est placée entre chevrons) : Dont clere est l'on $\langle d e\rangle$ et blanche la gravele. La musique de ces vers est écrite trois fois dans le manuscrit, elle est identique à chaque fois à un détail près : la musique du v. 2 dispose d'une note de plus, associée au - $e$ de la coupe épique. Comme indiqué plus haut, nous suivons l'indication du manuscrit, et considérons donc qu'il s'agit bien d'une coupe épique : dans l'onde et blanche, le - $e$ de l'onde est bien prononcé, mais il ne rend pas le vers faux, étant extramétrique. En Figure 3 sont représentées l'unique source musicale de cette chanson ainsi qu'une transcription de notre main. Sur le manuscrit, nous avons mis en évidence la différence entre la musique des v. 1 et 3 et celle du v. 2 qui nous intéresse. Dans la transcription, nous notons volontairement les élisions du - $e$ final par une apostrophe pour lever les ambiguïtés ; réciproquement, les - $e$ finaux qui sont écrits sont considérés prononcés.

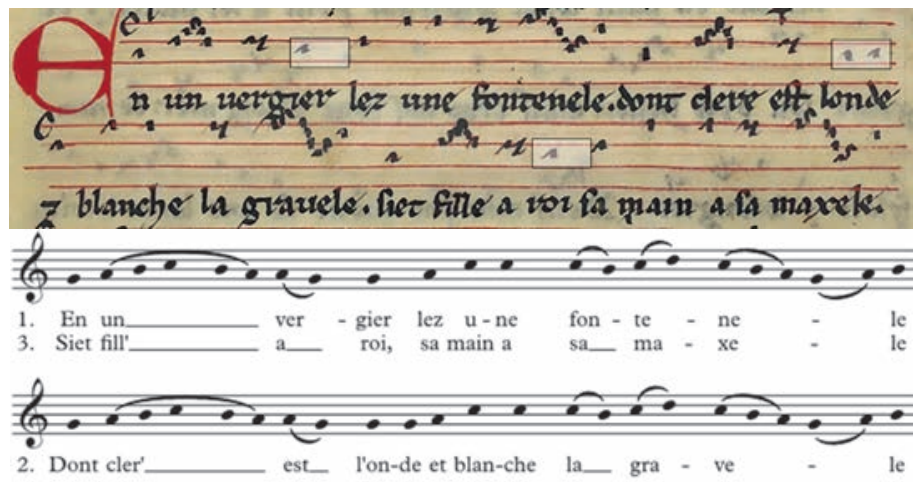

Figure 3. « En un vergier », vv. 1-3 (ms. U, fol 65v).

Dans cette chanson, le segment musico-textuel du v. 2 dispose d'une note de plus (sol, sur l'onde) que celui des vv. 1 et 3. En utilisant des crochets pour noter la constituance neumatique, il est possible d'avancer que la mélodie sol [la si do si la] [la sol] sol la do do etc. a été transformée en sol [la si do si la] [la sol] sol sol la do do etc. De fait, le contour mélodique sol la si do si la sol la do est 
inchangé, ce qui permet d’assurer l’identification de l'équivalence des segments musico-textuels.

Lorsque plusieurs manuscrits nous donnent la même chanson, l'emploi de cette stratégie de duplication n'est pas toujours constant. Ainsi, la chanson « La douce voiz du rosignol sauvage » du Châtelain de Coucy contient une coupe épique devant initiale vocalique au v. 7 : Si doi avoir grant joi $\langle e\rangle$ en mon corage, comme représenté en Figure 4. Huit manuscrits contiennent cette chanson, dont 5 ont, à quelques éléments près, la même musique : OMPX $a$. Les mss $\mathrm{OM}$ ignorent la coupe épique, tandis que PXa la reconnaissent et semblent la réaliser par une note.
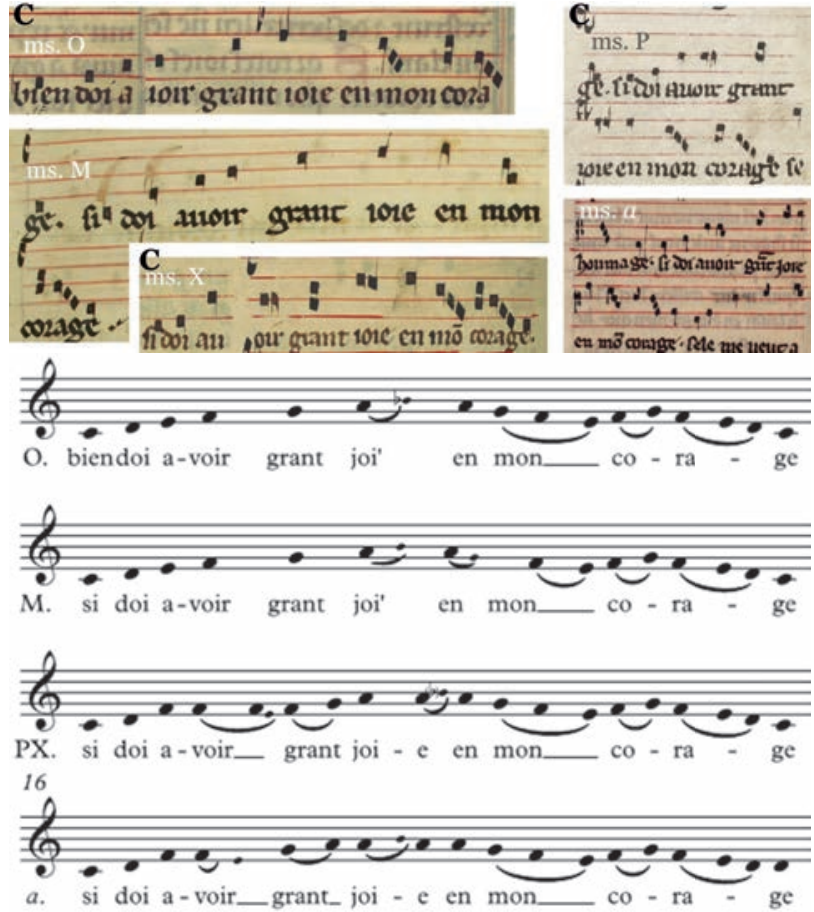

Figure 4. « La douce voiz du rosignol sauvage », v. 7

(mss O, fol. 74v ; M, fol. 54v ; P, fol. 33v ; X, fol. 71v; a, fol. 13r) ${ }^{16}$.

$\overline{16}$ Sur les mss, nous ajoutons des 'C' là où le montage a tronqué des clefs d'ut ; dans nos éditions, nous n'indiquons d’altérations que lorsque celles-ci sont présentes sur le manuscrit (à la clef ou devant la note), et ne les indiquons donc pas lorsqu'elles sont déductibles de la théorie de la musica ficta. 
Ainsi que représenté en Figure 4, OM pratiquent la synalèphe (ils n'ont pas de note sur laquelle chanter le $-e$ de joie, ce qui indique son élision : joi' en), tandis que les mss PXa proposent bien une note qui peut être associée au -e de joie. Ainsi, OM ne sont pas en infraction aux principes (4) $\sigma /$ Note et (5) Note/ $\sigma$, puisqu'ils ne prononcent pas le -e de joie. À l'inverse, PXa ajoutent une note, vis-à-vis de la musique de OM, puisqu'ils privilégient la coupe épique à la synalèphe. Dans les trois versions, un neume [la si] (au centre de la ligne dans notre transcription) est présent ; dans PX c'est ce neume qui reçoit le $-e$ de joie, tandis que dans $a$ ce neume est, comme pour OM, associé à la syllabe tonique joi-, et c'est après ce neume que se produit la duplication de $a$. La structure mélodique développée de chaque phrase est donnée en Figure 5 ; la seconde ligne ( $\forall$, 'pour tout') représente la leçon commune aux cinq manuscrits.

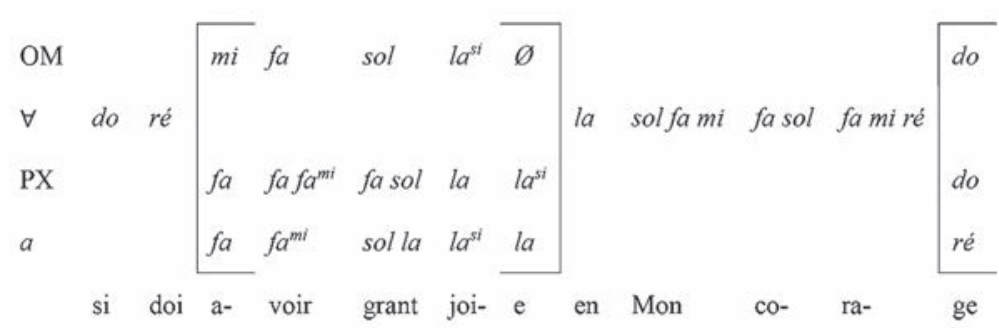

Figure 5. Structure mélodique du v. 7 de « La douce voiz du rosignol sauvage ».

Comme montré en Figure 5, les mss OMPXa partagent strictement la moitié de leur mélodie et de l'association entre notes et syllabes. La divergence de $a$ sur la note finale ne nous concerne pas (notre analyse ne porte pas sur cet élément) et nous la laissons donc de côté. Quant à la partie entre crochets sur avoir grant joie, nous pensons que les différences entre OM, PX et $a$ ne sont pas d'une première importance structurale. Dans les trois cas, ce passage peut se résumer à un contour mélodique fa sol la, le si étant placé dans une claire relation de dépendance vis-à-vis du la par l'usage de la plique (figure neumatique de dépendance entre deux notes). $\mathrm{Ce}^{\text {si }}$ n'est ainsi pas apte à recevoir de lui-même une syllabe, ce qui pour nous signifie qu'il ne participe pas à la structure du contour mélodique fondamental, mais à une étape plus développée, plus ornée, de la mélodie. Vis-à-vis de ce contour mélodique orné fa sol la $a^{s i}$, 
PX modifient l'alignement du texte ([la $\left.{ }^{\text {si }}\right]$ porte sur joi' dans OM, sur $-e$ dans PX), tandis que $a$ conserve $\left[l^{s i}\right]$ sur joi- et ne reproduit que la dans la duplication. La duplication, ici, ne cible donc pas des neumes entiers (sans quoi nous aurions $l a^{s i} l a^{s i}$ au lieu de $l a l a^{s i}$ et $\left.l a^{s i} l a\right)$, mais s'applique bien au niveau des notes qui composent les neumes. Ainsi, ce n'est pas le neume [ $\left.\mathrm{la}^{\text {si }}\right]$ qui entre en input de la musication, mais bien les notes dont il est composé, et en l'occurrence la seule note dotée d'un poids structural suffisant. En effet, ce n’est jamais le ${ }^{s i}$ qui est dupliqué ; il ne sert pas non plus à réaliser le - $e$ dans OM (la ligature du neume indique clairement qu'il porte sur la même syllabe que le la). Il semble donc que, au sein du contour mélodique fa sol $l a^{s i}$, toutes les notes ne sont pas égales dans leur capacité à recevoir une syllabe indépendante.

Le même phénomène est présent dans Aucassin et Nicolette, qui ne nous est parvenu que dans un seul ms. Ce récit de la fin du $\mathrm{XII}^{\mathrm{e}}$ siècle ou du début du XIII ${ }^{\mathrm{e}}$ siècle alterne régulièrement sections versifiées chantées et sections en prose récitées. Tous les passages versifiés sont chantés sur la même musique, composée de trois segments musicaux, probablement répétés selon une formule ABAB... $\mathrm{C}^{17}$. C étant uniquement écrit sur le vers orphelin final, plus court que les autres. La musique est présente sur le manuscrit pour chaque passage en vers, ce qui permet d'étudier sa variation entre les passages en vers féminins et les passages en vers masculins. La Figure 6 représente les premiers vers de trois passages chantés, l'un masculin (section VII, ms. à gauche), les deux autres féminins (III, ms. au centre, et v, ms. à droite ; pour ce dernier, seule la musique du premier vers est notée).

$\overline{17}$ La question de la formule d'itération des phrases A et B a fait l'objet d'un débat intense mais quelque peu aporétique ; comme chaque segment musical n'est écrit qu'une fois par passage versifié, cette question ne concerne pas notre étude. Le lecteur néanmoins curieux peut consulter ce qu'en dit Théodore Gérold dans RoQues, Mario (ed.), Aucassin et Nicolette, Chantefable du xIII siècle, Paris, Honoré Champion, 1925, pp. XXI-XXV, ainsi que VITZ, Evelyn Birge, "Variegated performance of Aucassin et Nicolette », en Eglal Doss-Quinby, Roberta L. Krueger, E. Jane Burns (eds.), Cultural performances in medieval France: Essays in honor of Nancy Freeman Regalado, Woodbridge, D.S. Brewer, 2007, pp. 235-245. 


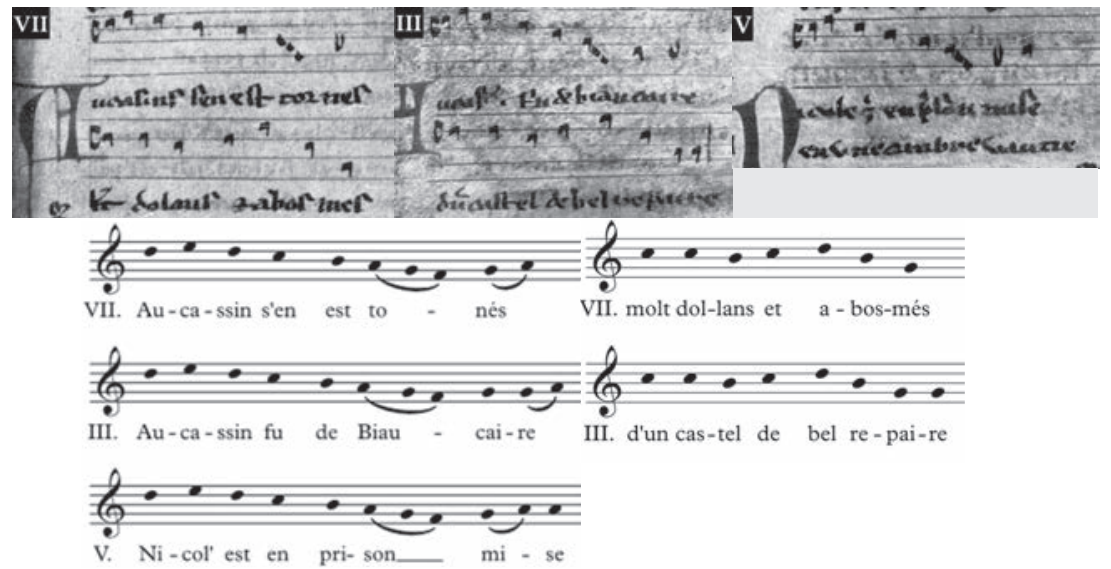

Figure 6. Aucassin et Nicolette, premiers vers des sections VII (gauche, fol. 72), III (centre, fol. 70v) et v (droite, fol. 71v).

Il convient d'observer ici le même phénomène de variation quant à la cible de la duplication. Pour le premier vers, face à un segment musico-textuel à rime masculine ré mi ré do si [la sol fa] [sol la], se trouvent deux segments musico-textuels à rime féminine : ré mi ré do si [la sol fa] sol [sol la] (III) et ré mi ré do si [la sol fa] [sol la] $l a(\mathrm{v})$, selon que c'est la première ou la seconde note du neume final qui a été ciblée par la duplication. Pour le second vers, exceptionnellement non recopié à la section v, sa duplication est plus stable : les sections féminines (sauf v, non notée) se terminent par si sol sol alors que les sections masculines se terminent par si sol ${ }^{18}$.

Enfin, ce fonctionnement se retrouve également dans Li Gieus de Robin et de Marion, texte de théâtre du XIII ${ }^{\mathrm{e}}$ composé par le trouvère Adam de la Halle, disposant de sections chantées dont les portées sont écrites directement sur le manuscrit au milieu du texte, comme pour Aucassin et Nicolette. Dans ce texte, se trouvent deux cas d'équivalence de segments musico-textuels masculins et féminins notés. Ces deux passages sont reproduits en Figure 7, dans la transcription proposée par Olivier Bettens ${ }^{19}$.

${ }^{18}$ Notons que la musique du v. 2 de la section Xxxv n'a pas la duplication alors qu'il s'agit bien d'un vers féminin. Il est impossible de ne pas considérer, ici, l'hypothèse d'une erreur de copie, d'autant que le caractère syllabique de la phrase musicale B (bijection syllabe-note) interdit toute possibilité de monnayer, comme disent les chanteurs, l'insertion de cette syllabe post-tonique au sein de ce segment musical.

19 De la Halle, Adam, « Li Gieus de Robin et de Marion », Olivier Bettens (ed.), 2002, en https://virga.org/robin/. 


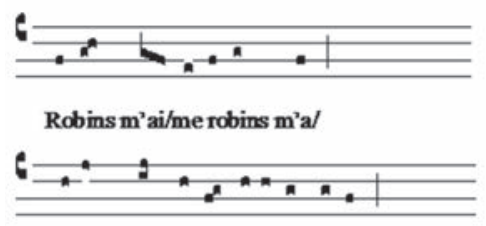

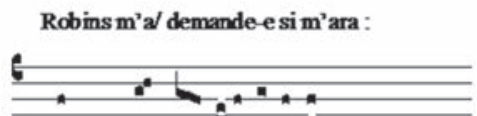

Ro- $[$ b] bins m'acata cole-le -

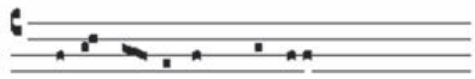

d'escarla- tebonne/ et bele

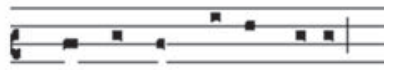

Hui main jou chevauchoie

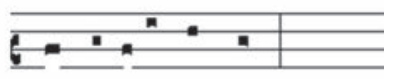

Kes l'o/riere d'umbois -

Figure 7. Li Gieus de Robin et de Marion, vv. 1-4 (colonne de gauche, fol. 39r) et 97-98 (colonne de droite, fol. 40r), dans la transcription diplomatique d'Olivier Bettens (pp. 14 et 19).

La comparaison de la musique du segment musico-textuel du v. 1 avec celle des segments musico-textuels des vv. 3 et 4, ainsi que celle du v. 97 avec celle du v. 98 illustre une fois de plus le même processus de duplication : la note finale du contour mélodique dans sa version masculine ( $f a$ au v. 1, ré au v. 98) a été dupliquée pour obtenir la version féminine ( $f a$ fa au vv. 3-4 et ré ré au v. 97).

À partir de ces exemples, et d'autres que nous n'illustrons pas ici puisqu'ils montrent le même type de configuration, il est possible de formaliser ainsi le fonctionnement de la duplication :

6. PARAmÈtres de LA DUPLiCATION ( $1^{\text {ère }}$ approximation)

a. La duplication prend pour input un segment musico-textuel A,

b. La duplication a pour output un segment musico-textuel A', différent de A,

c. La différence entre $A$ et $A^{\prime}$ tient au fait que $A^{\prime}$ dispose d'au moins une note et d'au moins une syllabe prononcée de plus que $\mathrm{A}^{20}$,

$\overline{{ }^{20}}$ La précaution au moins permet d'intégrer la circonstance où l'on aurait à adapter et la coupe épique et la terminaison féminine sur un même contour mélodique, et le fait qu'étant donnée la faible quantité des données disponibles, il n’est pas possible de prédire avec certitude que la duplication ne cible toujours qu'une seule note au sein d’un mélisme et non plusieurs notes, voire le mélisme entier. 
d. Au sein du contour mélodique, cette note supplémentaire dispose de la même hauteur qu'une note de A et est placée immédiatement après ou avant celle-ci.

7. Duplication et équivalence

a. La duplication a pour résultat de conserver le sentiment d'équivalence entre $A$ et $A$ ',

b. Elle ne tolère pas l'insertion d'une note dont la hauteur ne serait pas calquée sur une note située dans ses environs immédiats.

Le point (6)d ne contrevient pas à la conception du contour mélodique de Dell \& Halle ${ }^{21}$, dans la mesure où la répétition d'une hauteur de note ne modifie pas le contour mélodique. Sur la mélodie du premier vers d'Aucassin et Nicolette en Figure 6, que la duplication produise ... [sol fa mi] fa [fa sol] ou ... [sol fa mi] [fa sol] sol, le contour mélodique est toujours sol fa mi fa sol.

\section{III.2. Constituance neumatique et syllabicité}

La deuxième stratégie observable dans nos données, pour adapter le matériel musical à la variation de longueur du texte, consiste à modifier la constituance neumatique, c'est-à-dire la façon dont les notes sont regroupées en neumes. Comme nous l'avons montré dans la section précédente, la musication a accès aux notes qui constituent les neumes pour le procédé de duplication. La même capacité d'accès à l'information des notes par-delà leur constituance s'illustre également dans le fait que, lorsqu'un neume est disponible dans les environs immédiats du lieu où se trouve la variation de longueur du vers, l'ajustement de la musique à cette variation peut se faire en coupant le neume en deux sections, respectant ainsi les contraintes (4) $\sigma /$ Note et (5) Note/ $\sigma$ sans modifier aucunement le contour mélodique du segment musical.

La première illustration de ce phénomène se trouve dans la chanson « Bele Doette », dont les vv. 1 et 3 sont donnés en Figure 8. Notez, sur le manuscrit, que le mot amy est adscrit, ayant été probablement oublié par le scribe dans un premier temps. Ces deux vers sont chantés sur la même musique, au sein de la première strophe, mais le premier vers dispose d'une coupe épique devant initiale vocalique (Bele Doet $\langle t e\rangle$ as fenestres se siet) tandis que le second dispose d'une coupe masculine (De son amy Doon li resovient) ${ }^{22}$.

\footnotetext{
${ }^{21}$ Dell, François, \& Halle, John, « Comparing musical textsetting ... », cit., p. 69.

${ }^{22}$ Doette est trisyllabique : /do.c.tə/ ; Doon est bisyllabique :/do.ə̃n/.
} 


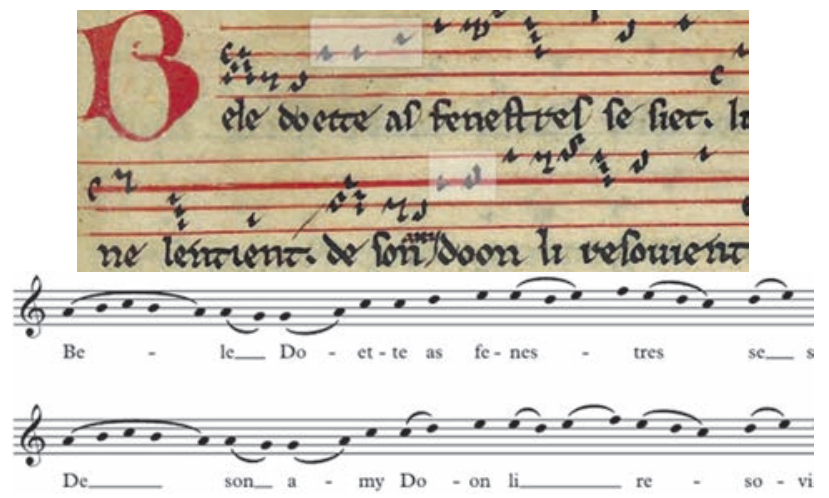

Figure 8. « Bele Doette », vv. 1 et 3 (ms. U, fol. 66r).

Comme le montre la Figure 8, le scribe a encore une fois privilégié la notation de la coupe épique à la synalèphe (ce qui est heureux, étant donné que dans d'autres strophes, la coupe épique se trouve devant une initiale consonantique qui n'autorise pas la synalèphe). Mais la différence entre les segments musico-textuels des vers 1 et 3 ne tient pas à une différence dans le nombre de notes, mais dans la constituance de celles-ci en neumes. Ainsi, à la coupe, sur Doette as et amy Doon, [sol la] do do ré au v. 1 s'oppose à [sol la] do [do ré] au v. 3. Cela ne modifie en rien le contour mélodique du segment musical, d'une part puisque que la répétition du do ne fait pas partie du contour, d'autre part parce que la modification de la constituance ne touche pas à la hauteur des notes.

Une autre illustration de ce phénomène peut être donnée avec la chanson "Amors qui m'a en baillie » du trouvère Jacques de Dosti. Dans cette chanson, les quatre premiers vers de la première strophe sont des vers de douze syllabes, césurés 7/5, et ils partagent presque la même musique selon qu'ils sont impairs ou pairs (itération musicale $\left.\mathrm{ABA}^{\prime} \mathrm{B}\right)$, mais les deux premiers vers ont une coupe épique devant initiale consonantique, tandis que les vv. 3 et 4 ont des coupes masculines ${ }^{23}$ :

8. Amors, qui m’a en bailli $\langle\mathrm{e}\rangle$, veut qu’envoissié soie ; Je ferai chançon joli〈e $\rangle$, puis qu'ele l'otroie. Puis que ma dame a mon cuer, drois est qu'a li soie ; $\quad \mathrm{A}^{\prime}$ S’el ne me veut recevoir, jamés n’avrai joie.

A $\mathrm{B}$

$\overline{{ }^{23}}$ Notons que seule la première strophe fait rimer ses syllabes toniques à la coupe, ce qui valide l'analyse en vers de 12 syllabes métriques; il ne faut donc pas analyser ces hémistiches comme étant des vers entiers. 

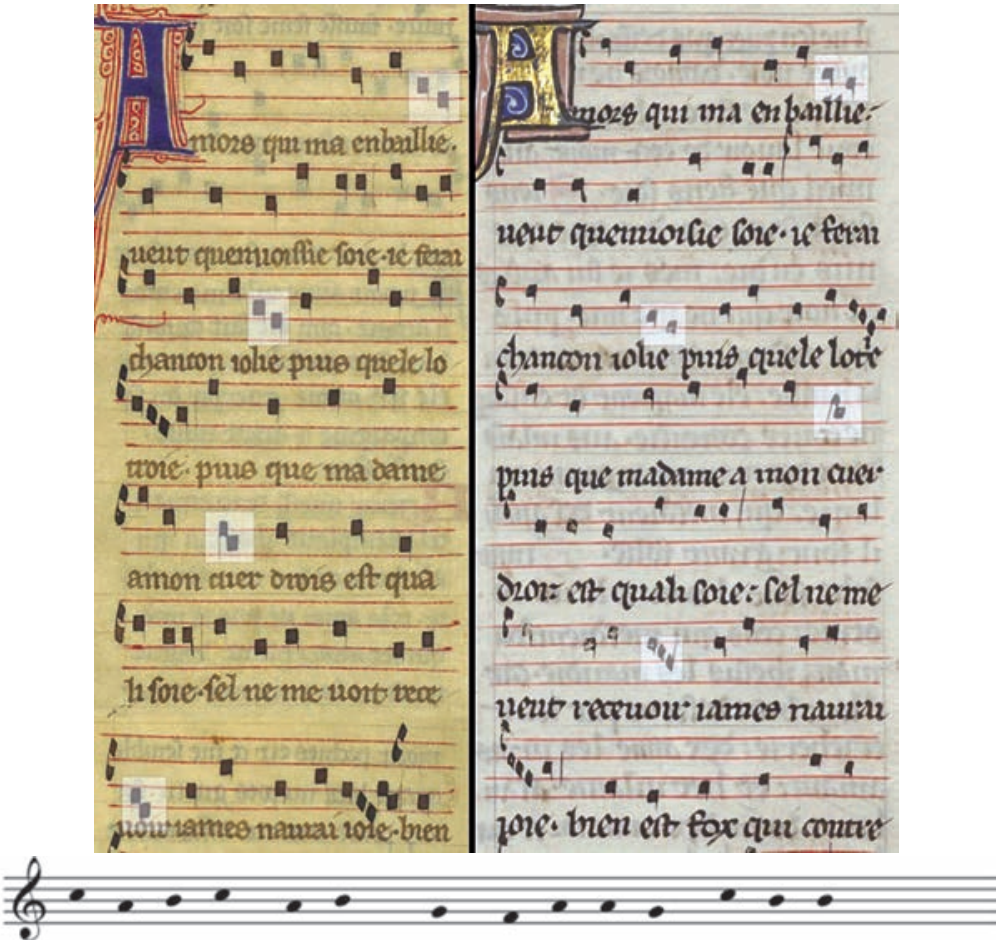

1. A-morsqui m'a en bai - lli - e veutqu'envoi - sié soi - e

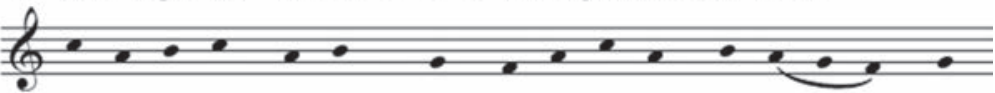

2. Je fe-rai chan-son jo - li - e puisqu'e-le l'o-troi - e

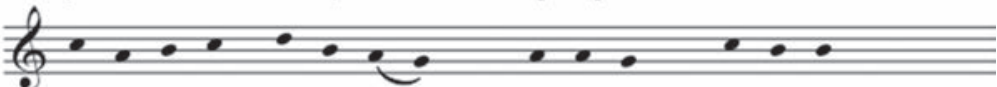

3. Puisque madam' a mon cuer_ drois estqu'a li soi-e

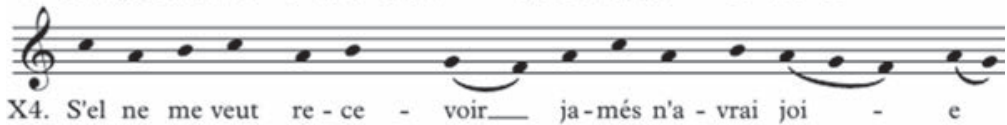

X4. S'el ne me veut re-ce - voir_ ja-més n'a - vrai joi - c

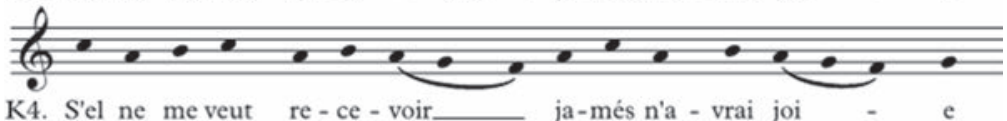

Figure 9. "Amors qui m'a en baillie »,

vv. 1-4 (mss X, fol. 238v (gauche) et K, p. 365 (droite)). 
Les vv. 1 et 3 ont une musique très proche mais qui dispose de variations non négligeables, ne se limitant pas au lieu de la coupe épique qui nous intéresse (puisque la s’oppose à ré à la cinquième note). Même si A et A' semblent être des variantes d'un même segment musical, il nous est difficile d'établir rigoureusement le point à partir duquel un segment musical donné cesse d'être équivalent à un autre segment musical pour devenir indépendant. Aussi nous nous concentrerons plutôt sur ce qui se produit aux vv. 2 et 4, plus immédiatement semblables. Le v. 2, dont la leçon est identique dans les deux manuscrits, réalise la coupe épique en respectant (4) $\sigma /$ Note et (5) Note/ $\sigma$, le mot jolie étant chanté sur si sol fa. Au v. 4 , sol et fa sont bien présents dans nos deux manuscrits (sur -voir de recevoir), mais ils sont liés dans un neume, ce qui permet de respecter (5) NotE/ $\sigma$ puisque les deux notes sont bien associées à une syllabe. Remarquons également, dans $\mathrm{K}$, la présence d'une note supplémentaire, probablement ornementale (c'est une 'note de passage'), mais qui ne vient pas modifier fondamentalement la mélodie. Cette note de passage appartient également au neume qui regroupe les deux notes non liées au v. 2. Remarquons enfin un autre type d'ornementation à la fin du v. 4 dans X, qui a [la sol fa] la sol là où $\mathrm{K}$ a [la sol fa] sol, l'insertion d'un la supplémentaire ne venant évidemment pas modifier l'équivalence fondamentale entre les deux segments musicaux. Cela montre, comme attendu, que ces variations, de manuscrits en manuscrits ou de segments en segments au sein de la même pièce et dans le même témoin, ne se produisent pas uniquement sur le lieu de l'adaptation du matériel mélodique à la variation de longueur du vers.

Alors que pour la duplication c'était le contour mélodique du segment musico-textuel masculin qui était considéré comme plus proche du contour mélodique fondamental sous-jacent, avec l'opposition entre notes liées en un neume et notes unitaires, il n'est pas possible de déduire quelle structure est la plus proche de ce contour fondamental, d'autant que celui-ci ne comprend en principe, tel que formulé par Dell \& Halle, que les hauteurs de notes. Ainsi, à ce niveau fondamental, la différence entre [la sol] et la sol n'existe pas. Comme la question de la syllabicité n’appartient pas à ce contour mélodique fondamental, il apparaît qu'en analyse formelle ce n'est pas la musication du vers avec coupe féminine qui est dérivée de celle du vers avec coupe masculine, mais que les deux dérivent de la même structure, non sensible à la constituance 
neumatique, indifféremment [la sol] et la sol. Il ne s’agit donc pas d'une dérivation de type [la sol] $\rightarrow$ la sol, qui briserait un neume pour respecter (4) $\sigma$ /Note, ou d'une dérivation la sol $\rightarrow$ [la sol] qui regrouperait en un neume deux notes syllabiques pour respecter (5) Note/ $\sigma$, mais d'une optimisation naturelle du lien entre notes et syllabes, à partir d'un niveau de contour mélodique non spécifié en constituance syllabique. Il est possible de formaliser ainsi ce phénomène :

9. Spécification de la syllabicité

a. La spécification de la syllabicité prend pour input un contour mélodique A non structuré en constituants syllabiques et un segment textuel,

b. La spécification de la syllabicité a pour output un contour mélodique A', structuré en constituants syllabiques et donc associés aux syllabes du texte, dont la constituance est spécifiée en fonction du nombre de syllabes et des contraintes (4) $\sigma /$ Note et (5) Note/ $\sigma$.

10. Spécification de la syllabicité et équivalence

a. La spécification de la syllabicité a pour effet de respecter le sentiment d'équivalence entre les différences instances de $\mathrm{A}$, même si celles-ci diffèrent dans leur regroupement de notes en constituants syllabiques.

Enfin, il faut apporter une dernière précision : dans toutes nos chansons, c'est bien la constituance neumatique qui entre en jeu, et non la constituance en mélismes. Cela est surprenant, dans le sens où la propriété importante, ici, est celle de l'association de plusieurs notes avec une seule syllabe, propriété commune aux neumes et aux mélismes. Un mélisme pouvant intégrer un ou plusieurs neumes, il apparaît que le neume est un constituant hiérarchiquement inférieur au mélisme. Les principes (9) et (10) devraient donc pouvoir s'appliquer à tous les mélismes et non simplement aux neumes, mais dans nos données, ce sont toujours des neumes qui en font l’objet. Nous avons donc choisi de rédiger (9) et (10) en conformité avec nos données, de la manière la plus restrictive possible ; nous ne voyons cependant pas d'objections à ce que (9) et (10) s'appliquent directement à des mélismes dans d'autres chansons. 


\section{3. Reformulation de la duplication}

La formalisation de la duplication que nous avons proposée en (6) était rédigée dans une visée de surface, selon laquelle le matériel musical du segment musico-textuel de surface A était dérivé de manière à s’associer à un segment textuel plus long, donnant ainsi le segment musico-textuel A', les deux segments étant équivalents ${ }^{24}$. Nous avons vu que, dans le cas de la spécification de la syllabicité, il n'était pas approprié de définir quel segment de surface était l'input de la dérivation, et quel segment de surface en était l'output, mais que cette difficulté était aisément contournée si nous admettons que les deux réalisations de surface étaient toutes deux des instanciations équivalentes d'un contour mélodique fondamental A. La différence entre les deux instanciations vient de l'association de A avec deux textes dont le nombre de syllabes est différent. Ces adaptations sont motivées par le respect des conditions (4) б/NotE et (5) Note/ $\sigma$. Nous pouvons donc réécrire la formalisation des paramètres de la duplication comme suit :

11. Duplication :

a. La duplication prend pour input un contour mélodique $\mathrm{A}$, sans répétitions de note à hauteur constante, et un segment textuel,

b. La duplication a pour output un contour mélodique A', associés aux syllabes du texte, dont les répétitions à hauteur constante sont spécifiées en fonction du nombre de syllabes et des contraintes (4) $\sigma /$ NoTE et (5) Note/ $\sigma$,

12. Duplication et ÉQuivalence :

a. La duplication a pour effet de respecter le sentiment d'équivalence entre les différences instances de A, même si celles-ci diffèrent dans leurs répétitions de notes à hauteur constante.

$\overline{{ }^{24}}$ Sur l'existence, dans d'autres contextes musicaux, d'une telle homologie de surface, voir Premat, Timothée, Chouvion, Sophie, \& Verner, Axelle, « Repetitions, rests insertion and Schwa in $16^{\text {th }}$ century French Polyphony: An Emergence Sub-Grammar in Fresneau's Songs? », en Michela Russo (ed.), The Emergence of Grammar: A Closer Look at Dialects between Morphology and Phonology, New York, Nova, [à paraître]. 


\section{IV. Équivalence et localité des adaptations}

Ces deux processus, duplication et spécification de la syllabicité, permettent donc d'adapter un segment musical unique à des vers de longueur différente, tout en conservant la reconnaissance de l'équivalence de ces différents segments musicaux. Cette équivalence se fait par la reconnaissance d'une forme sous-jacente commune, que nous avons provisoirement identifiée au contour mélodique. Une dernière chose doit néanmoins être précisée : dans aucun des cas d'adaptation que nous avons trouvés, le contraste de nombre de notes ou de constituance syllabique n'apparaît à distance de la localisation de la syllabe extramétrique. C'est-à-dire que l'ajustement du matériel mélodique à la variation de longueur de vers ne se produit que là où cette variation de longueur se produit. Nous n’avons pas trouvé, par exemple, de cas de duplication ou d'opposition de constituance syllabique qui se produirait à la toute fin d'un segment musico-textuel alors que la coupe épique se trouve en son milieu. Or la mécanique produite par la combinaison de (4) $\sigma /$ Note, (5) Note/ $\sigma$, (9) SpécificAtion de la Syllabicité et (11) DuPLICATION ne propose pas de clause de localité.

Cette clause de localité est difficile à exprimer en surface, et ce principalement pour deux raisons. La première raison tient au fait que (9) SpÉCIFICATION DE LA SYLlABICITÉ et (11) DuPLICATION peuvent être progressives ou régressives, ce qui fait qu'il n'est pas possible de prédire positivement quel segment du contour mélodique va être adapté à la présence d'une syllabe extramétrique. La deuxième difficulté tient au fait que (9) SPÉCIFICATION DE LA SYLLABICITÉ et (11) DuPLICATION sont déclenchées par une syllabe qui, en surface, n’a rien de dissociable : seul le statut métrique du - $e$ de la coupe épique et de la rime féminine est spécifique et déclenche (9) et (11). Or ce statut métrique n'est pas présent en surface, mais peut être déduit à partir d'une échelle de représentation sous-jacente : le gabarit métrique.

Ces difficultés peuvent être contournées par une définition négative de la localité. Cette définition se fonde sur le fait que, en surface, l'équivalence entre différents segments musico-textuels tend à une forme de parallélisme entre eux, et ce à différents niveaux ${ }^{25}$. Nous proposons que ce parallélisme entre deux segments musicaux et

${ }^{25}$ Dell, François, « Répétitions parallèles dans les paroles et dans la musique des chansons ", en Jean-Louis Aroui (ed.), Le sens et la mesure. De la pragmatique à la métrique. Hommages à Benoît de Cornulier, Paris, Champion, 2003, pp. 499-522. 
textuels implique également une distribution parallèle du lien entre syllabes et notes. Dans une optique de dérivation d'une forme sousjacente vers une forme de surface, il faut donc stipuler qu'il existe une association entre un niveau de contour mélodique et un niveau de représentation du texte. Ce niveau de représentation du texte doit être le dénominateur commun entre les vers à coupe masculine et ceux à coupe épique, et entre les vers à rime masculine et ceux à rime féminine; il s’agit donc de leur patron métrique, constitué d'une suite de positions ${ }^{26}$. Ce niveau de représentation musico-textuelle n'est pas encore spécifié en termes de syllabicité, mais il l'est en termes d'association entre notes et positions. Nous l'appelons donc niveau musico-positionnel. Ce niveau est illustré en Figure 10, sur la musique des vv. 1 et 3 de la chanson « Bele Doette » (cf. Figure 8 supra). Nous représentons les positions métriques par des ' $\mathrm{X}$ ', sans nous préoccuper ni des frontières du vers ou des hémistiches, ni de la proéminence des positions. Des principes analogues à (4) $\sigma /$ NoTE et (5) NoTE/ $\sigma$ contraignent cette association entre notes et positions.

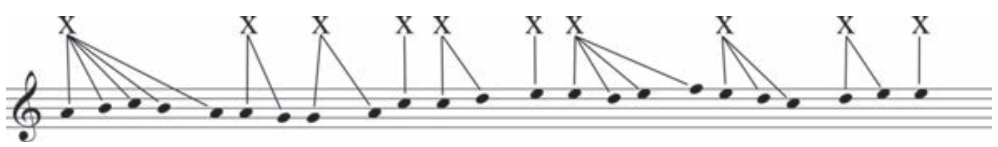

Figure 10. « Bele Doette », association entre notes et positions, vv. 1 et 3.

Cette association entre notes et positions est, par définition, commune à tous les vers qui ont le même gabarit métrique et qui sont associés au même segment musical. Elle est donc commune aux vers 1 et 3 . Par la suite, ce segment musico-positionnel est

${ }^{26}$ Dans une optique générative-gabaritique de la métrique, une position est l'équivalent abstrait d'une syllabe métrifiée. Un ensemble de positions forme le gabarit métrique d'un type de vers donné, et des règles détaillent les associations possibles entre positions et syllabes. De ces associations résultent des vers, dotés de leur contenu linguistique. Pour l'analyse gabaritique des vers français, voir Verluyten, S. Paul, « L'analyse de l'alexandrin, Mètre ou rythme ? », en Marc Dominicy, (ed.), Le souci des apparences : neuf études de poétique et de métrique, Bruxelles, Éditions de l’Université de Bruxelles, 1989, pp. 31-74 ; Dell, François, \& Benini, Romain, La Concordance chez Racine. Rapports entre structure grammaticale et structure métrique dans le théâtre de Racine, Paris, Classiques Garnier, [à paraître] ; Premat, Timothée, Chouvion, Sophie, \& Verner, Axelle, "Repetitions, rests insertion and Schwa... ", cit. ; Chouvion, Sophie, Premat, Timothée, \& Verner, Axelle, « La musication dans les chansons d'Henry Fresneau, Grammaire d'association, paraphonologie et forme polyphonique au xvie siècle ", [ms. soumis à publication dans les actes des IVèmes rencontres franco-italiennes Philologie et Musicologie (Morimondo/Pavie, 1-5/06/2019) dans la revue Textus \& Musica, soumis en 2020]. 
associé aux syllabes d'un vers donné, et c'est là que s'appliquent (9) SpÉcification de la Syllabicité et (11) Duplication. Avec une coupe épique ou une rime féminine, l'adjonction du texte au niveau musico-positionnel crée des syllabes flottantes : les syllabes extramétriques ne sont rattachées à aucune note, ce qui crée une infraction à (4) $\sigma /$ Note si (5) Note/ $\sigma$ est respecté. Cette infraction est réparée en ré-assignant une ou plusieurs notes du niveau musico-positionnel à la syllabe flottante ((9) SPÉCIFICATION DE LA SYLLABICITÉ) ou en redoublant l'une des notes du niveau musico-positionnel pour l'associer à la syllabe flottante ((11) DuPLICATION).

Dans notre exemple, c'est (9) SPÉCIFICATION DE LA SYLLABICITÉ qui entre en jeu. Au v. 3, il y a autant de syllabes que de positions, donc l'association entre notes et syllabes est naturellement identique à l'association entre notes et positions. Mais, au v. 1, il y a une syllabe surnuméraire, ce qui crée une tension entre (4) $\sigma /$ NoTE et la volonté de parallélisme entre l'association notes-positions et l'association notes-syllabes. La solution déployée par notre manuscrit consiste alors à briser le parallélisme, en associant la onzième note (deuxième do au milieu de la ligne) avec la syllabe extramétrique, comme illustré en Figure $11^{27}$. Cela n'est possible que parce que plusieurs notes étaient associées à la position métrique environnante ; sinon, la duplication ou la synalèphe (qui neutralise le déclenchement de (4) $\sigma$ /NotE) se seraient substituées à (9) SPÉcIFICATION DE LA SYLLABICITÉ.
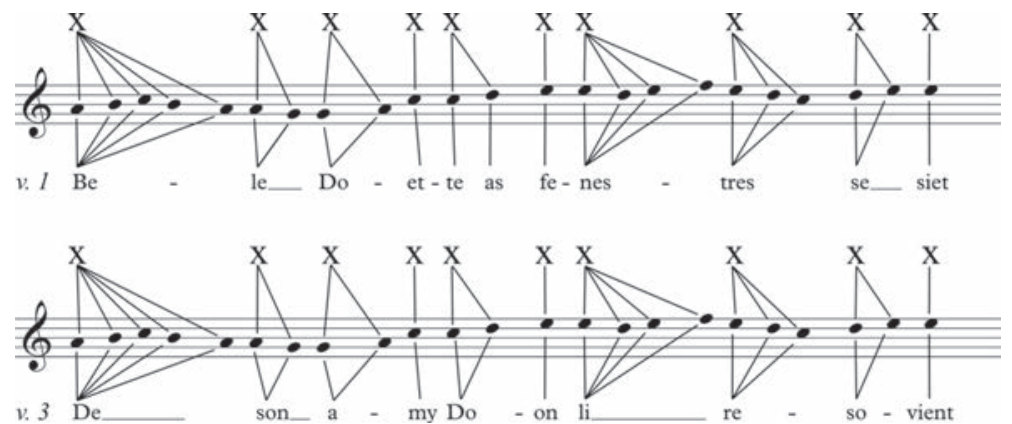

Figure 11. « Bele Doette ", associations entre notes et positions et entre notes et syllabes, vv. 1-3.

$\overline{27}$ Le lien entre syllabes et positions n’est pas représenté pour des questions de lisibilité du schéma, mais il est sous-entendu : toutes les syllabes sauf le -e de Doette sont associées à la position placée au-dessus d'elles. 
Si cela ne se fait que dans l'environnement immédiat de la syllabe flottante, c'est parce qu'appliquer (9) SPÉCIFICATION DE LA SYLLABICITÉ ou (11) DuPLICATION à un autre endroit du segment musico-positionnel provoquerait plus de changements dans les liens entre notes et positions, alors que son application locale permet de modifier le moins possible ces liens. La localité, comprise comme transparence maximale entre association notes-positions et association notes-syllabes, est donc nécessaire au maintien de l'équivalence.

Nous proposons donc la formalisation suivante :

13. Notes\&Positions. La première étape d'association entre musique et texte est structurale ; elle distribue les notes en fonction des positions du gabarit métrique du texte. Elle doit respecter les contraintes (14-15) :

14. Position/Note. Toute position doit être associée à au moins une note.

15. Note/Position. Toute note doit être associée à une et une seule position.

16. Notes\&Syllabes. À l'issue de (13), la seconde étape d'association entre musique et texte distribue les notes en fonction des syllabes d'un vers donné. Elle doit respecter les contraintes (4)-(5) :

(4) $\sigma /$ NotE. Toute syllabe prononcée doit être associée à au moins une note.

(5) NoTE/ $\sigma$. Une note doit être associée à une et une seule syllabe.

17. Équivalence. Pour que deux segments musico-textuels A' et A" soient équivalents, ils doivent apparaître comme deux instanciations du même segment musico-positionnel A. Pour cela, A' et A" doivent répondre aux conditions suivantes :

a. Conservation du Contour Mélodique. A' et A" doivent avoir le même contour mélodique,

b. Conservation de L'Association Notes\&Positions. A' et A" doivent avoir la même association entre notes et positions (13),

c. Transparence Notes\&Positions - Notes\&SYlLABES. Dans chaque terme de l'équivalence (A' et A'), le lien entre notes et syllabes doit être le plus proche possible du lien entre notes et positions. 
Ainsi, la tension entre d'une part (4) $\sigma /$ Note et (5) Note/ $\sigma$, et d'autre part (17b), impose, sans que soit modifiée l'association entre notes et positions issues de (13) Notes\&Positions, une modification du matériel musical ou de son association au texte qui ne consiste pas en un changement de hauteur de note (pour respecter (17a)). Cette modification s'opère au niveau local de manière à minimiser les modifications entre l'output de (13) Notes\&Positions et celui de (16) NoTES\&SYLLABES, la condition de transparence (17c) étant nécessaire à la perception de la communauté de NoTES\&PoSiTIONs (13) entre les segment musico-textuels. (9) SPÉCIFICATION DE LA SYLLABICITÉ et (11) DuPLICATION représentent donc des solutions optimales pour adapter la succession des notes aux variations de longueur du texte, en maximisant la transparence entre l'output de (13) et celui de (16) tout en respectant les contraintes (14)-(15), (4)-(5) et (17)).

\section{Conclusion}

Pour cette étude, nous nous sommes intéressés aux adaptations du matériel musical aux variations de longueur du texte dans un corpus de musique vocale en langue d'oïl des XII $^{\mathrm{e}}$ et $\mathrm{XIII}^{\mathrm{e}}$ siècles. Pour cela, nous avons sélectionné des pièces musicales (a) dotées de segments musicaux équivalents entre eux, (b) dont les segments musicaux équivalents sont associés à des vers disposant d'une variation de longueur et (c) dont les segments musico-textuels soient écrits au moins deux fois, une pour chaque longueur de vers.

Sur cette base, nous avons documenté les différentes adaptations du matériel musical dont témoignent nos manuscrits. Celles-ci sont principalement de deux ordres : la duplication et la spécification de la syllabicité. Nous avons établi les paramètres de ces deux dérivations, dans une optique de dérivation depuis une structure sous-jacente commune aux segments musico-textuels sans syllabes extramétriques et à ceux qui disposent d'au moins une syllabe extramétrique.

Enfin, nous avons remarqué que ces dérivations ont un caractère local, mais que celui-ci n’est pas aisé à établir sous forme de relation entre les formes de surface. Cela est en revanche aisé à établir si nous admettons un niveau sous-jacent d'association entre les notes et les positions métriques du texte. Ce niveau d'association entre notes et positions doit ensuite être dérivé en association entre notes et syllabes, et nous espérons avoir montré en quoi la duplication et 
la spécification de la syllabicité étaient deux solutions optimales pour respecter les différentes contraintes qui pèsent sur l'association entre notes et syllabes. Elles ont en effet pour conséquence la conservation de caractéristiques idoines à la reconnaissance d'une relation d'équivalence entre les segments musico-textuels.

\section{Sources manuscrites}

a, Rome, Biblioteca Vaticana, Reg. 1490, https://digi.vatlib.it/view/MSS_Reg.lat.1490.

K, Paris, BNF, Bib. de l'Arsenal, Ms-5198, https:/gallica.bnf.fr/ark:/12148/ btv1b550063912.

M, Paris, BNF, fr. 844, https://gallica.bnf.fr/ark:/12148/btv1b84192440.

O, Paris, BNF, fr. 846, https://gallica.bnf.fr/ark:/12148/btv1b6000950p.

P, Paris, BNF, fr. 847, https://gallica.bnf.fr/ark:/12148/btv1b8454673n.

U, Paris, BNF, fr. 20050, https://gallica.bnf.fr/ark:/12148/btv1b60009580.

X, Paris, BNF, nouv. acq. fr. 1050, https://gallica.bnf.fr/ark:/12148/btv1b530003205.

Aucassin et Nicolette, Paris, BNF, fr. 2168, fol. 70r-80v, https://gallica.bnf.fr/ark:/12148/ btv1b9058947p.

Li Gieus de Robin et de Marion, Paris, BNF, fr. 25566, fol. 39r-48v, https://gallica.bnf.fr/ ark:/12148/btv1b6001348v. 